\title{
WIDELY CONNECTED AND BICONNECTED SEMIGROUPS
}

\author{
PAUL M. SWINGLE
}

We have shown in [7] that under certain assumptions both widely connected and biconnected topological semigroups exist. As point sets both of these sets have peculiar properties including the fact that the only continua either can contain are degenerate connected sets; this gives contrast to the well known result by Pontrjagin that a bicompact connected topological group contains an arc; also R. J. Koch has shown recently [2] that a bicompact connected topological semigroup with unit also contains an arc; our semigroups here are obviously not bicompact. Below we develop some further properties of these as semigroups.

We assume that we have a topology under which $S$ is a nondegenerate connected semigroup; this involves a continuous associative multiplication which is a mapping $m: S \times S \rightarrow S$. We recall from [8, p. 254] that $S$ is widely connected if and only if every nondegenerate connected subset has the same closure that $S$ has. We need below that $m$ be extendable to $\bar{S}$, the closure of $S$, and that $\bar{S}$ be a compact perfectly separable space: we will also take it as a Hausdorff one. Our first results deal with the properties that $m$ must have if $S$ is a widely connected semigroup and our last if $S$ is a biconnected semigroup with dispersion point, recalling from $[1, \mathrm{p} .214]$ that $S$ is biconnected if and only if it is not the union of two disjoint nondegenerate connected subsets; and $d$ is a dispersion point of $S$ if and only if $S-d$ is totally disconnected.

We write $\varnothing$ for the null set. We mean by $M-N$ the set $\{p \mid p \in M$ but $p$ non $\in N\}$. For basic definitions see Moore in [3] and Wallace in [4]. We recall, for $A, B \subset S$, then $A B=m(A \times B)$ $=\{m(a, b) \mid a \in A, b \in B\}=\{a b \mid a \in A, b \in B\}$. If $x \in S$ and $x^{2}=x$, then $x$ is an idempotent of $S$ and $E=\left\{x \mid x^{2}=x, x \in S\right\}$. If $\varnothing \neq N \subset S$, then $N$ is a left ideal if $S N \subset N ; N$ is a right ideal if $N S \subset N ; N$ is an ideal if it is both a left and a right ideal. Below by $A, B, C$ and $D$ we always mean the following:

$$
\begin{aligned}
& A=\{a \mid a S \text { is a point, } a \in S\}, \quad B=\{b \mid S b \text { is a point, } b \in S\} \\
& C=\{c \mid c \bar{S} \supset S, c \in S\} \quad \text { and } \quad D=\{d \mid \bar{S} d \supset S, d \in S\} .
\end{aligned}
$$

Presented to the Society, August 27, 1958 under the title Widely connected algebras; received by the editors September 16, 1958 and, in revised form, May 20, 1959. 
LEMмA 1. If $S$ is a semigroup and if $a S$ and $S b$ are both points for some $a, b \in S$, then $S b=a S=a^{2}=b^{2}$ and $a S$ is a minimal ideal of $S$.

Proof. If $a S$ is a point for some $a \in S$, then $a S=a a=a^{2}$ and similarly $S b=b^{2}$. Also $S S b \subset S b=b^{2}$ and so $S b$ is a left ideal and obviously minimal. Similarly $a S$ is a minimal right ideal. Since it is a known and easily proved property that each left and right ideal intersect, we see that $a S=S b=a^{2}$ and so $a^{2}$ is a minimal ideal of $S$.

Lemma 2. If $S$ is a topological semigroup and $A \neq \varnothing \neq B$, then $A$ and $B$ are each closed in $S$ and $S B=A S=a^{2} \in S$.

Proof. Since $A \neq \varnothing \neq B$, from $\left(1^{*}\right)$ we see that there exist $a \in A$ and $b \in B$ satisfying Lemma 1 and so $a S=S b=a^{2}$ and $a S$ is a minimal ideal of $S$. If $a^{\prime} \in A$, since each left and right ideal intersect, we see that $a^{\prime} S=a S$. Thus it follows that $A S=S B=a^{2}$. It easily follows that $A$, and similarly $B$, is closed for under the supposition that it is not, one gets a contradiction with the fact that $m: S \times S \rightarrow S$ is a continuous mapping.

Lemma 3. If $S$ is a topological semigroup, then $C$ and $D$ are each closed in $S$.

Proof. It easily follows that $C$, and similarly that $D$, is closed for under the supposition that there exists $c_{0} \in S-C$ and $c_{0}$ is a limit point of $C$ we have that $c_{0} \bar{S} \supset S$ is false by definition $\left(1^{*}\right)$ of $C$. For a fixed $s \in S$ there exist $c_{i} \in C$ for $(i=1,2, \cdots)$ and $c_{i}^{\prime} \in \bar{S}$ such that $c_{i} c_{i}^{\prime}=s$, because from $\left(1^{*}\right) c_{i} \bar{S} \supset S$. Then, since $\bar{S}$ is compact and perfectly separable, there exists $c_{0}^{\prime} \in \bar{S}$ such that $c_{0} \times c_{0}^{\prime}$ is a sequential limit of a subsequence of $\mathrm{U}\left(c_{i} \times c_{i}^{\prime}\right)$; it follows that $c_{0} c_{0}^{\prime}=s$ and so $c_{0} \bar{S} \supset S$, which is a contradiction.

LEMMA 4. If $S$ is a widely connected semigroup, then, for each $a, b \in S$, $a S$ is either a point or $a \bar{S} \supset S$; similarly $S b$ is either a point or $\bar{S} b \supset S$.

Proof. Since $S$ is connected, it is known and easy to prove that $a S$ and $S b$ are both connected. By definition of widely connected then $a S$ is either a point, i.e. a degenerate connected set, or $S$ and $a S$ have the same closure, i.e. (closure of $a S)=\bar{S} \supset S$. It follows without difficulty from the fact that $m: S \times S \rightarrow S$ is a continuous mapping that (closure of $a S$ ) $=a \bar{S}$. Thus either $a S$ is a point or $a \bar{S} \supset S$ and similarly either $S b$ is a point or $\bar{S} b \supset S$.

Lemma 5. If $S$ is a videly connected semigroup and $A \neq \varnothing \neq B$, then $C=\varnothing=D$. 
Proof. By Lemmas 2 and 3, $A$ and $C$ are each closed; by Lemma 4, $S=A \cup C$ and so $S$ cannot be connected unless $C=\varnothing$. Similarly for $D$.

Theorem 1. If $S$ is a widely connected semigroup then one of the following cases must be true: (1) $S=A=B$, i.e. $S=A \cap B$; (2) $S=A=D$, i.e. $S=A \cap D$; (3) $S=B=C$, i.e. $S=B \cap C$; (4) $S=A \cup C=D$ where $A \neq \varnothing$, i.e. $S=A \cup(C \cap D)$; (5) $S=B \cup D=C$ where $B \neq \varnothing$, i.e. $S=B \cup(C \cap D)$; or (6) $S=C=D$, i.e. $S=C \cap D$.

Proof. By Lemma 4 for $a \in S$ either $a \in A$ or $a \in C$. Thus $S=A$ or $S=C$ or $S=A \cup C$. Similarly for $b \in S$ either $b \in B$ or $b \in D$. Thus $S=B$ or $S=D$ or $S=B \cup D$. Thus for $S=A$ we can have (1) $S=B$ or (2) $S=D$, but by Lemma 5 we cannot have $S=A \neq \varnothing$ and $S=B \cup D$ where $B \neq \varnothing \neq D$. Also for $S=C$ we can have (3) $S=B$ or (6) $S=D$ or (5) $S=B \cup D$. For $S=A \cup C$ we cannot have $A \neq \varnothing \neq B$ and $C \neq \varnothing$ and so cannot have $S=B$ by Lemma 5 ; we can have (4) $S=D$ but cannot have $S=B \cup D$ unless it is one of the other cases. Hence we conclude that the Theorem is true.

Corollary 1.1. In cases (1)-(3) of Theorem 1 we have $S^{2} \subset E$, where $E$ is the set of idempotents of $S$.

Proof. Consider as typical the cases (1) and (2) where $S=A$. Let $a \in A$, i.e. $a S=a^{2}$ by $\left(1^{*}\right)$. Let $a b \in S^{2}$. Then $a b=a^{2}$; also $a(b a b)=a^{2}$. Therefore $a b=a^{2}=(a b)^{2}$ and so $a b \in E$ and hence $S^{2} \subset E$.

Lemma 6. Let $S$ be a semigroup and $a \in S$. Then $a^{2}$ is a right zero of $S$ if $S a$ is a point; also $a^{2}$ is a right zero of $S$ only if $S a$ is a point, if also, for each $s \in S$, there exists some $b \in S$ such that $b a=s$.

Proof. Consider the case where $S a$ is a point. Then $S a=a^{2}$. For each $b \in S$ there exists $s=b a \in S$; then $(b a) a=a^{2}=b a^{2}$. Hence $a^{2}$ is a right zero of $S$.

Consider the case where $a^{2}$ is a right zero of $S$. Then, for $b \in S$, $b a^{2}=a^{2}$. Hence $S a=a a$, for if $s \in S$ there exists $b \in S$ such that $b a=s$.

COROLlaRy 1.2. If $S$ is a widely connected semigroup, then for every $a \in S$ we have $a^{2}$ is either a zero, a left zero or a right zero of $S$ in cases (1), (2) or (3) respectively of Theorem 1.

Proof. That $a^{2}$ is a right zero in case (3) follows at once from Lemma 6; in case (2) a similar lemma would show that $a^{2}$ is a left zero; the combination of these gives that $a^{2}$ is both a left and a right zero, i.e. a zero, of $S$ in case (1).

We remark that the widely connected semigroup of which we 
showed the existence in [7], being a topological group, was case (6) of Theorem 1 as Lemma 6 shows.

THEOREM 2. If $S$ is a widely connected semigroup, then the minimal ideal $K$ of $S$ always exists in cases (1)-(5) of Theorem 1; in cases (1) $-(3) K=S$ and in cases (4)-(5) $\bar{K}=\bar{S}$; in case (6) each left and each right ideal of $S$ is dense in $S$.

Proof. We showed in the proof of Lemma 1 that $a \in A$ implies that $a^{2}$ is a minimal right ideal, i.e. an m.r.i., and that $b \in B$ implies that $b^{2}$ is a minimal left ideal, i.e. an m.l.i.; it is known [6, Lemma 19] that the minimal ideal $K$ is the union of the m.r.i. and also the union of the m.1.i.; thus it follows that $K$ exists in cases (1)-(5) because either $A \neq \varnothing$ or $B \neq \varnothing$. Thus also in (1)-(3) we have $K=S$ because either $A=S$ or $B=S$.

Consider (4) where $S=A \cup(C \cap D)$. By Lemma $3 C$ and $D$ are each closed in $S$ and so $C \cap D$ is closed in $S$. Since $S$ is connected, $A \neq \varnothing$ must contain at least two points. Thus $K$ contains these by the above Lemma 19 of [6]; $K$ is known to be connected and so it is not degenerate connected. Hence by definition of widely connected we see $\bar{K}=\bar{S}$ in case (4) and similarly in case (5).

In case (6) $S=D=C$. For $d \in S$ we have $S \supset S d$ and for $d \in D$ by $\left(1^{*}\right)$ we have $\bar{S} d \supset S$. Hence $\bar{S} d=\bar{S}$. Let for example $L$ be a left ideal of $S$ and $d \in L$. Then $S d \subset S L \subset L \subset S$. Thus from $\bar{S} d=\bar{S}$ we have $\bar{L}=\bar{S}$. Similarly for right ideals of $S$.

Lemma 7. If $S$ is a semigroup then $A, B, C$ and $D$ are all semigroups.

Proof. Let $c, c^{\prime} \in C$. Then $c \bar{S} \supset S$, by $\left(1^{*}\right)$, and so $c^{\prime} c \bar{S} \supset c^{\prime} \bar{S} \supset S$. Therefore, by $\left(1^{*}\right), c^{\prime} c \in C$ and so $C$ is a semigroup as similarly is $D$. Let $a, a^{\prime} \in A$. Then $a S=a a$, by $\left(1^{*}\right)$, and so $a^{\prime} a S=a^{\prime} a a$ which is a point. Therefore $a^{\prime} a \in A$ by $\left(1^{*}\right)$ and so $A$ is a semigroup as similarly is $B$.

Lemma 8. Let $S$ be a semigroup. Then for each $c \in C$ there exist $c^{\prime \prime}, e^{\prime \prime} \in \bar{S}$ such that $c e^{\prime \prime}=c$ and $c c^{\prime \prime}=e^{\prime \prime} ;$ and for $d \in D$ there exist $d^{\prime}$ and $e^{\prime} \in \bar{S}$ such that $e^{\prime} d=d$ and $d^{\prime} d=e^{\prime}$.

Proof. For $c \in C$ by $\left(1^{*}\right)$ we have $c \bar{S} \supset S \supset c$. Therefore there exists $e^{\prime \prime} \in \bar{S}$ such that $c e^{\prime \prime}=c$. Also $c \bar{S} \supset \bar{S} \supset e^{\prime \prime}$ and so there exists $c^{\prime \prime} \in \bar{S}$ such that $c c^{\prime \prime}=e^{\prime \prime}$. Similarly for $d \in D$ there exist $d^{\prime}$ and $e^{\prime}$ as desired.

Lemma 9. Let $S$ be a semigroup and $f \in C \cap D$. Then there exists $e \in \bar{S}$ such that $f e=f=e f$, i.e. $e$ is a unit for $f$.

Proof. By Lemma 8 there exist $e^{\prime}, e^{\prime \prime}, f^{\prime}$ and $f^{\prime \prime}$ such that $e^{\prime} f=$ 
$f=f e^{\prime \prime}$ and $f^{\prime} f=e^{\prime}$ and $f f^{\prime \prime}=e^{\prime \prime}$. Then $e^{\prime} e^{\prime \prime}=f^{\prime} f e^{\prime \prime}=f^{\prime} f=e^{\prime}$ and $e^{\prime} e^{\prime \prime}=$ $e^{\prime} f f^{\prime \prime}=f f^{\prime \prime}=e^{\prime \prime}$. Hence $e^{\prime}=e^{\prime \prime}$, $=e$ say. Thus $f e=e=e f$.

THEOREM 3. Let $S$ be a widely connected semigroup, $E$ be the set of idempotents of $S$ and $F=\left\{f \mid f \in S\right.$ and there exist $e, f^{\prime}, f^{\prime \prime} \in \bar{S}$ such that $\left.f e=f=e f, f^{\prime} f=e=f f^{\prime \prime}\right\}$. Then $C \cap D,=F$, is a semigroup and either $S^{2} \subset E, S=F$, or $S=F \cup E^{\prime}$ where each $a \in E^{\prime}$ is such that $a a \in E$.

Proof. In cases (1)-(3) of Theorem 1 we see by Corollary 1.1 that $S^{2} C E$. By Lemmas 8 and 9 we have that $F=C \cap D$. By the proof of Lemma 7 we see that $C \cap D$ is a semigroup. In case (6) $S=F$. In cases (4)-(5) we have that $S=F \cup E^{\prime}$ by Lemma 6 .

We note that a widely connected set $S$ is a hereditarily indecomposable connected set and also $S$ is closed in itself. Thus the results above and also Theorem 1 of [7] hold for a nonbicompact, hereditarily indecomposable continuum which is a topological semigroup $S$; here $S$ can be a topological group.

In the following $S$ is a biconnected topological semigroup with a dispersion point $d$. Since $S a$ is a connected subset of the biconnected set $S$, it is biconnected and has dispersion point $d$ if it is nondegenerate. To obtain the results below we put on conditions limiting to a point the subset of $S$ which maps into $d$ by $S a$, where $a \neq d$.

Lemma 10. Let $S$ be a biconnected semigroup with dispersion point $d$; furthermore, for $a \in S$ and $a \neq d$, let there exist $Z \subset S$ such that $Z a=d$; also, if $Z a=d$, let then $Z$ be a point. Then either aa is a right zero of $S$ or $d$ is a left zero for $a$.

Proof. Consider the case where $a a$ is not a right zero of $S$. Then, by Lemma $6, S a$ is not a point. Therefore it follows from the definition of biconnected that $S a$ is biconnected and has dispersion point $d$. Hence $S a-d=S_{1}^{\prime} \cup S_{2}^{\prime}$, where $S_{1}^{\prime}$ and $S_{2}^{\prime}$ are mutually separate. If $s^{\prime} \in S_{i}^{\prime} \subset S a,(i=1,2)$, then there exists $s \in S$ such that $s a=s^{\prime}$. Let $S_{i} a=S_{i}^{\prime}$. Then, for $Z a=d$ of the hypothesis, $S a-d=S a-Z a=S_{1} a$ $\cup S_{2} a$ separate. If $s_{0} \in S_{2}$ and it is the sequential limit of $s_{j} \in S_{1}$, $(j=1,2, \cdots)$, then $s_{0} a$ is the sequential limit of the $s_{j} a$. We conclude that $S-Z=S_{1} \cup S_{2}$, where $S_{1}$ and $S_{2}$ are mutually separate. By hypothesis $Z$ is a point and so $Z=d$, since a biconnected set can have but one dispersion point. Thus $Z a=d a=a$ and so $d$ is a left zero for $a$.

Lemma 11. Let $S$ and $Z$ be as in Lemma 10 and

$$
G=\{g \mid g \in S-d, d g=d\} .
$$

Then $G \cup d$ is closed in $S, S=(G \cup d) \cup B$ and $G \cap B=\varnothing$. 
Proof. Let $g_{j} \in G,(j=1,2, \cdots)$, have sequential limit $g_{0}$. Then $d g_{j}=d$ has sequential limit $d g_{0}$ and so $g_{0} \in G \cup d$. Therefore $G \cup d$ is closed in $S$.

Suppose that $g \in G \cap B$. Then $S g=g g=d g=d$ by (1*) and hypothesis. For $Z=d \cup g, Z g=d$ and $Z$ is not a point. Hence $G \cap B=\varnothing$.

Let $H=\{h \mid h \in S-d, d=h d\}$. Then by proofs similar to those of Lemma 10 and Lemma 11 we have without difficulty:

THEOREM 4. Let $S$ be a biconnected semigroup with dispersion point $d$. Let $x \in S-d$ and $Z, Z^{\prime} \subset S$ exist such that $Z a=d=a Z^{\prime}$; further, if $Z x=d$, then $Z$ is a point and, if $x Z^{\prime}=d$, then $Z^{\prime}$ is a point. Then one of the following must be true: (1) $S=A=B$; (2) $S=A=G \cup d$; (3) $S=B$ $=H \cup d ; \quad$ (4) $S=H \cup d=(G \cup d) \cup B$ where $B \neq \varnothing ;$ (5) $S=G \cup d$ $=(H \cup d) \cup A$ where $A \neq \varnothing$; or (6) $S=H \cup d=G \cup d$. Thus, for every $s \in S$, ss is a zero of $S$, or $d$ is either a left zero, a right zero or a zero of $S-d$.

The biconnected semigroup we give in [7] is an example of case (6), $d$ being a zero for $S$.

\section{REFERENCES}

1. B. Knaster and C. Kuratowski, Sur les ensembles connexes, Fund. Math. vol. 2 (1921) pp. 206-253.

2. R. J. Koch, Arcs in partially ordered spaces, to appear.

3. R. L. Moore, Foundations of point set theory, Amer. Math. Soc. Colloquium Publications, vol. 13, 1932.

4. A. D. Wallace, The structure of topological semigroups, Bull. Amer. Math. Soc. vol. 61 (1955) pp. 95-112.

5. A. D. Wallace and R. J. Koch, Maximal ideals in compact semigroups, Duke Math. J. vol. 21 (1954) pp. 681-685.

6. - Notes on mobs, Tulane University, mimeographed, 1956.

7. P. M. Swingle, Existence of widely connected and biconnected semigroups, Proc. Amer. Math. Soc. vol. 11 (1960) pp. 243-248.

8. — , Two types of connected sets, Bull. Amer. Math. Soc. vol. 37 (1931) pp. $254-258$.

University OF Miami 